\title{
Tecnura
}

http://revistas.udistrital.edu.co/ojs/index.php/Tecnura/issue/view/640

DOI: http://dx.doi.org/10.14483/udistrital.jour.tecnura.2014.SE1.a10

INVESTIGACIÓN

\section{Evaluación de los protocolos MAC IEEE 802.11 y MMAC-CR en una red MANET}

\section{Evaluation of MAC IEEE 802.11 and MMAC-CR protocols within a MANET}

\author{
Judy C. Guevara*, Luis Fernando Pedraza Martínez**, César Augusto Hernández Suárez*** \\ Citation / Para citar este artículo: Guevara, J., Pedraza Martínez, L. F., \& Hernández Suárez, C. A. (2014). Evaluación \\ de los protocolos MAC IEEE 802.11 Y MMAC-CR en una red MANET. Revista Tecnura, Edición especial, 125-134.
}

Fecha de recepción: 14 de junio de 2012 / Fecha de aceptación: 9 de noviembre de 2013

\section{RESUMEN}

Este artículo presenta un análisis comparativo entre los mecanismos de control de acceso al medio IEEE 802.11 y MMAC-CR (Multichannel MAC protocol for Cognitive Radio) en redes MANET (Mobile Ad Hoc Networks). El estándar IEEE 802.11 permite el uso de múltiples canales disponibles en la capa física, pero su protocolo MAC está diseñado para un solo canal. Sin embargo, un protocolo MAC de un solo canal no trabaja bien en un entorno multicanal debido al problema del terminal oculto. La metodología del artículo se basa en la descripción de los protocolos de control de acceso al medio, la simulación del throughput de estos, el análisis de los resultados y las conclusiones. Los resultados de simulación muestran cómo el protocolo MMAC-CR permite hacer un mejor uso de las oportunidades espectrales, incrementando de esta manera el throughput de la red MANET.

Palabras clave: radio cognitiva, control de acceso al medio, redes ad hoc, protocolo MAC multicanal, redes inalámbricas.

\begin{abstract}
This paper presents a comparative analysis between the mechanisms associated to Media Access Control (MAC) IEEE 802.11 and MMAC-CR (Multichannel MAC protocol for Cognitive Radio) in MANETs (Mobile Ad Hoc Networks). The IEEE 802.11 standard allows the use of multiple channels available at the physical layer, but its MAC protocol is designed for a single channel. However, a single channel MAC protocol does not operate properly in a multi-channel environment due to the hidden terminal problem. The methodology of the article is based on the description of MAC protocols, including throughput simulations, results analysis and some conclusions. The simulation results show how the MMAC-CR protocol allows a better use of spectral opportunities, thereby increasing throughput on the MANET network.
\end{abstract}

Keywords: Cognitive radio, medium access control, ad hoc networks, multi-channel MAC protocol, wireless networks.

\footnotetext{
* Ingeniera en control, magíster en ciencias de la información y las comunicaciones. Docente, Universidad Manuela Beltrán. Bogotá, Colombia. Contacto: judy.guevara@docentes.umb.edu.co

** Ingeniero electrónico, magíster en ciencias de la información y las comunicaciones; candidato a doctor en ingeniería de sistemas y computación de la Universidad Nacional de Colombia. Docente, Universidad Distrital Francisco José de Caldas. Bogotá, Colombia. Contacto: Ifpedrazam@udistrital.edu.co

*** Ingeniero electrónico, magíster en ciencias de la información y las comunicaciones; candidato a doctor en ingeniería de sistemas y computación, de la Universidad Nacional de Colombia. Docente, Universidad Distrital Francisco José de Caldas. Bogotá, Colombia. Contacto: cahernandezs@udistrital.edu.co
} 


\section{INTRODUCCIÓN}

Durante las dos últimas décadas la experiencia de los usuarios orientada a entornos colaborativos en tiempo real ha hecho de la movilidad un aspecto predominante en todas las tecnologías y dispositivos de comunicaciones modernos. Así mismo, existen situaciones en las que se requiere un rápido despliegue en una zona sin infraestructura o se debe establecer un sistema de comunicación en áreas colapsadas en donde las comunicaciones fijas han sido averiadas. Ante estas circunstancias resultan ideales redes sin infraestructura como las MANETs, formadas por dispositivos móviles que se conectan a través de enlaces inalámbricos compartidos, y que además deben crear rutas multi-salto cuando la fuente y el destino no están dentro de sus rangos de transmisión (Koes, 2004). Estas redes presentan algunas características poco deseables, como el ancho de banda reducido que debe ser compartido por todos los nodos que componen la red. El estudio de este problema puede ser abordado desde dos perspectivas diferentes. La primera ha sido empleada tradicionalmente y corresponde al estándar IEEE 802.11. La segunda responde a un esquema novedoso, el protocolo MMAC-CR (De Domenico, 2012), usado en radio cognitiva (CR) para establecer múltiples canales en paralelo, asegurando de esta forma un uso eficiente de todas las oportunidades espectrales.

Este artículo presenta un análisis comparativo del comportamiento de una red MANET cuando implementa en su capa MAC los protocolos IEEE 802.11 y MMAC-CR, cuyos fundamentos se explican brevemente en la sección II. En la sección III se describe el entorno de simulación en el cual se evaluaron estos dos protocolos. La sección IV expone los resultados obtenidos de la simulación. La discusión de los resultados se encuentra en la sección V. El artículo finaliza con las conclusiones que aparecen en la sección VI.

\section{FUNDAMENTOS}

En esta sección se da a conocer el principio de operación de los protocolos IEEE 802.11 y MMACCR (Guevara, 2013).

\section{Función de coordinación distribuida de IEEE 802.11}

Inicialmente el objetivo de agregar múltiples canales a IEEE 802.11 era mejorar el desempeño de las redes con infraestructura. Pero en la actualidad este protocolo también es usado por las redes ad hoc, en cuyo interior se emplea un mismo canal para la comunicación de todos los nodos, independientemente de si se encuentran o no dentro del rango de transmisión de uno de sus vecinos (Bahl, 2004). El estándar IEEE 802.11b, por ejemplo, emplea la técnica de Espectro Ensanchado por Secuencia Directa (DSSS) y provee una capa física con 14 canales, separados en frecuencia $5 \mathrm{MHz}$. Sin embargo, para evitar que se traslapen, la separación en frecuencia debe ser al menos de $30 \mathrm{MHz}$. De manera que en la práctica, son usados los canales 1, 6 y 11 en la banda de $2.4 \mathrm{GHz}$, tal como lo indica la figura 1.

La operación del protocolo IEEE 802.11 se basa en la Función de Coordinación Distribuida (DCF), en la cual un nodo reserva un canal para la transmisión de datos a través del intercambio de mensajes RTS (Ready to Send)/CTS (Clear to Send) con el nodo objetivo. Cuando un nodo desea transmitir paquetes a otro nodo, debe enviar primero un paquete RTS al destino. El receptor responde enviando un paquete CTS al transmisor. Tanto el paquete RTS como el CTS incluyen el tiempo estimado durante el cual el canal estará en uso. Los demás nodos que escuchen estos paquetes deben posponer su transmisión durante el tiempo especificado en los paquetes. Por esta razón cada nodo mantiene una variable llamada NAV (Network Allocation Vector) que graba el tiempo por el cual debe posponerse su transmisión (So, 2004). Este proceso en general se conoce como detección virtual de 
portadora, y permite reservar el área alrededor del transmisor y el receptor para la comunicación, evitando así el problema del terminal oculto.

La figura 2 ilustra la operación de la DCF de IEEE 802.11. Cuando el nodo B está transmitiendo un paquete al nodo $C$, el nodo $A$ escucha el paquete RTS y envía su NAV hasta el final del $A C K$, mientras que el nodo $D$ escucha el paquete CTS y configura su NAV hasta el final del ACK.
Tan pronto como se completa la transmisión, las estaciones esperan durante un intervalo de tiempo Ilamado DIFS (DCF Interframe Space) y luego compiten por el canal. En esta figura, el nodo B es un terminal oculto para el nodo D. Sin una detección virtual de canal, el nodo $\mathrm{D}$ puede desconocer la transmisión del nodo $\mathrm{B}$ y puede transmitir un paquete a $\mathrm{C}$ mientras $\mathrm{B}$ está transmitiendo, produciendo una colisión a C.

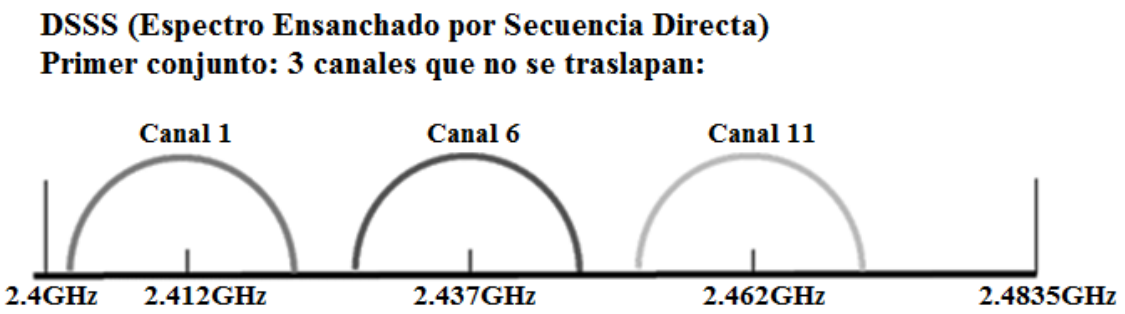

DSSS (Espectro Ensanchado por Secuencia Directa)

Segundo conjunto: 6 canales que se traslapan a la mitad

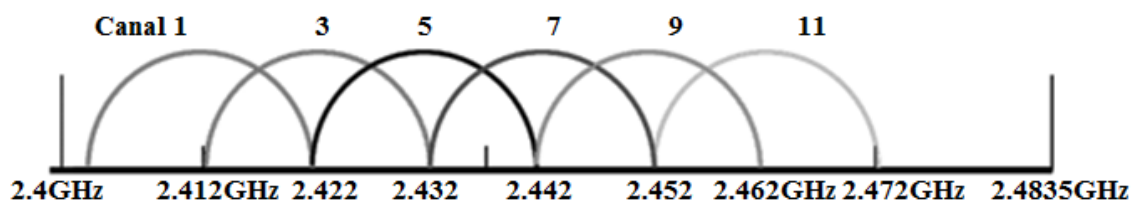

Figura 1. Canales del estándar IEEE 802.11b.

Fuente: Tomado de FCC (2012).

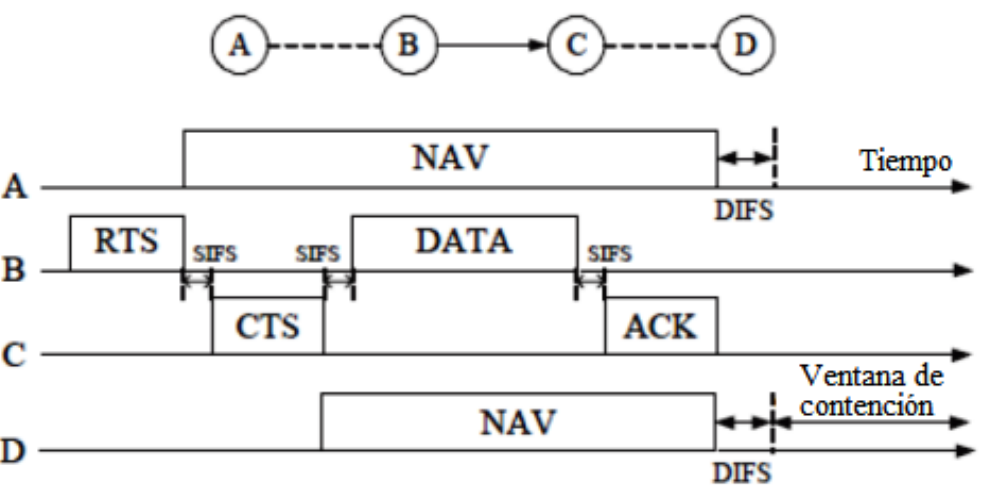

Figura 2. Operación de la DCF de IEEE 802.11.

Fuente: Tomado de So (2004). 
Si un nodo tiene que enviar un paquete pero observa que el canal está ocupado, este escoge un contador de backoff no mayor que un intervalo llamado ventana de contención (CW), que corresponde a una variable que tiene cada nodo. Esta variable es reseteada al valor CWmin cuando el nodo es inicializado y también luego de cada trasmisión exitosa. Después de escoger el valor del contador, el nodo esperará hasta que el canal esté libre, y luego decrementará el contador. El contador es decrementado en uno luego de cada "slot de tiempo", siempre y cuando el canal esté libre. Si el canal es ocupado, el nodo debe congelar el contador hasta que el canal sea liberado. Debido a que dos nodos pueden tomar el mismo contador de backoff, el paquete RTS puede perderse por una colisión. Dado que la probabilidad de colisión aumenta con el incremento del número de nodos, un transmisor debe interpretar la ausencia de un CTS como una señal de congestión. En este caso el nodo doblará su ventana de contención para reducir la probabilidad de otra colisión.

Antes de transmitir un paquete, todo nodo tiene que esperar un tiempo corto Ilamado espacio intertrama, aun cuando el canal esté libre. Existen cuatro intervalos de tiempo diferentes que habilitan cada paquete según su prioridad, en orden de menor a mayor longitud son SIFS (Short Interframe Space), PIFS (Point Coordination Function Interframe Space), DIFS y EIFS (Extended Interframe Space). Por ejemplo, un nodo espera un DIFS antes de transmitir un RTS, pero espera un SIFS antes de enviar un CTS o un ACK. Así, un ACK puede ganar el canal cuando compite con un RTS o un paquete de datos porque la duración de un SIFS es menor que la de un DIFS.

\section{MAC Multicanal para radio cognitiva}

En este protocolo cada CR mantiene dos estructuras de datos, una es el vector de imagen espectral de usuario primario (SIP) y la otra es el vector de carga de canal de usuarios secundarios (SCL). El vector SIP[n] representa la estimación del uso del espectro del canal c, y puede contener los siguientes valores (Timmers, 2010):

- Cuando no hay PU activo sobre el canal C, SI$\mathrm{P}[\mathrm{c}]=0$.

- Cuando un PU está activo sobre el canal C, SI$\mathrm{P}[\mathrm{c}]=1$.

- Cuando la presencia de un PU es incierta, SIP[C] $=2$.

Cuando un nodo se une a la red, realiza un escaneo rápido para cada canal en la ventana ATIM (Ad Hoc Traffic Indication Message). El resultado de ese escaneo es almacenado en el vector SIP. Después del sensado inicial, los valores del vector SIP son actualizados usando los valores del escaneo. El vector SIP es usado para determinar si la red puede usar un determinado canal para la comunicación de datos. Además se emplea para determinar si el nodo requiere programar un nuevo escaneo durante el período de datos, el cual es necesario cuando el valor de SIP de un canal es incierto.

Por otra parte, el vector SCL se usa para seleccionar el canal de comunicación. Este contiene la carga esperada de comunicación CR en cada canal. Cuando un nodo desea transmitir, escoge la oportunidad espectral con SCL el más bajo.

La figura 3 muestra la estructura de tiempo que maneja este protocolo. El tiempo es dividido en intervalos guía de longitud fija en los cuales se distinguen dos fases: la ventana ATIM y la ventana de datos. Durante la ventana ATIM, los nodos realizan un escaneo rápido e intercambian información de control. Durante la ventana de datos tiene lugar el intercambio de datos y el sensado fino.

También durante la ventana ATIM, los nodos conocen las oportunidades espectrales existentes en toda la red escuchando los minislots de C. El protocolo de minislots es inicializado luego de la transmisión o recepción del paquete SRP (Scan Result Packet) que contiene el resultado del escaneo sobre el canal de control. La función de este paquete es asegurar una estrecha sincronización siguiendo la función de sincronización (TFS) de IEEE 802.11. 


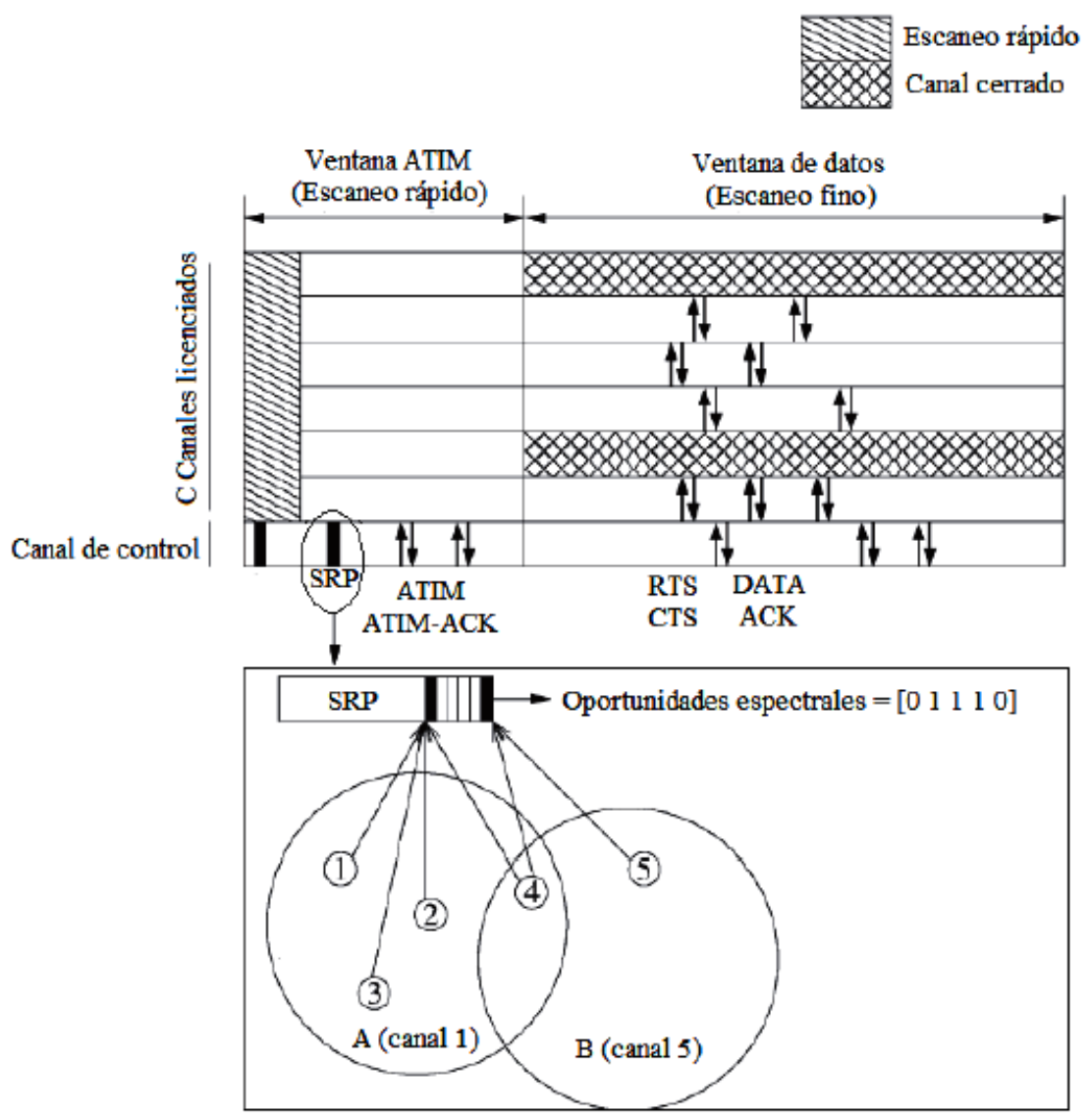

Figura 3. Escaneo y comunicación en paralelo sobre diferentes canales. En el ejemplo los nodos 1-5 aprenden que dos PNs (A y B) están activos sobre los canales 1 y 5.

Fuente: Tomado de Timmers (2010).

Si un minislot es sensado como ocupado, a través de un valor de SIP diferente a cero, el canal correspondiente es excluido de la comunicación CR. Los nodos que tienen paquetes almacenados indican el tráfico enviando tramas ATIM sobre el canal de control durante la ventana ATIM. En la trama ATIM, un nodo inserta el canal de preferencia para la transmisión, por ejemplo, el que tenga el SCL más bajo y el estado de su cola. Cada nodo que escuche la trama ATIM actualizará su vector SCL. Si el nodo receptor está de acuerdo con el canal seleccionado, responde con una trama ATIM-ACK. Luego de la ventana ATIM, los nodos que han intercambiado tramas ATIM permanecerán activos hasta que se haya completado el intercambio de datos. Los nodos que no transmitieron o no recibieron tramas ATIM entran en un estado de reposo hasta el siguiente intervalo guía.

Durante la ventana de datos no solo se realiza el intercambio de datos sino que además, los nodos que tienen configurado el SIP como incierto efectúan un escaneo fino del canal correspondiente. Este puede ser hecho en paralelo con la comunicación sobre otro canal. Luego, se actualiza el valor SIP para este canal. El intercambio de datos sigue el procedimiento normal de la DCF de IEEE 802.11 con el intercambio RTS/ 
CTS. Una característica adicional es que se permite a los nodos entrar en un estado de reposo cuando completan el intercambio en la ventana de datos, por ejemplo si la cola de transmisión está vacía.

\section{ENTORNO DE SIMULACIÓN}

La influencia de los protocolos MAC multicanal en el comportamiento de la red MANET fue evaluada mediante la herramienta de simulación ns-2.31 (UC Berkeley, 2012). En la tabla 1 se especifican los parámetros con los cuales se caracterizó la red.

Tabla 1. Parámetros de simulación.

\begin{tabular}{ll}
\hline \multicolumn{1}{c}{ Parámetros } & \multicolumn{1}{c}{ Valor } \\
\hline Área & 350 X $400 \mathrm{~m}$ \\
\hline No. de nodos & 6 \\
\hline Modelo de movilidad & Random Waypoint \\
\hline Velocidad de los nodos & $5 \mathrm{~m} / \mathrm{s}$ \\
\hline Tiempo de pausa & $15 \mathrm{~s}$ \\
\hline Interfaz de red & Phy/Wireless Phy \\
\hline Modelo de propagación & Tworayground \\
\hline Tipo de antena & Ominidireccional \\
\hline Protocolo de encaminamiento & AODV \\
\hline Protocolo de la capa de transporte & TCP \\
\hline Tamaño de los paquetes & 512 bytes \\
\hline Duración & $50 \mathrm{~s}$ \\
\hline
\end{tabular}

Fuente: Elaboración propia.

Con el fin de simular el comportamiento de los nodos que componen la red se seleccionó el modelo de movilidad Random Waypoint. En este modelo, los nodos parten de una posición inicial determinada dentro del área asignada y a medida que avanza el proceso de simulación realizan movimientos en zig-zag de manera aleatoria, como lo ilustra la figura 4. Además, cada nodo hace una pausa en la ubicación en que se encuentra para amortiguar los cambios abruptos de dirección. Los movimientos individuales de los nodos fueron generados a través de la herramienta BonnMotion v1.5a (BonnMotion, 2012).

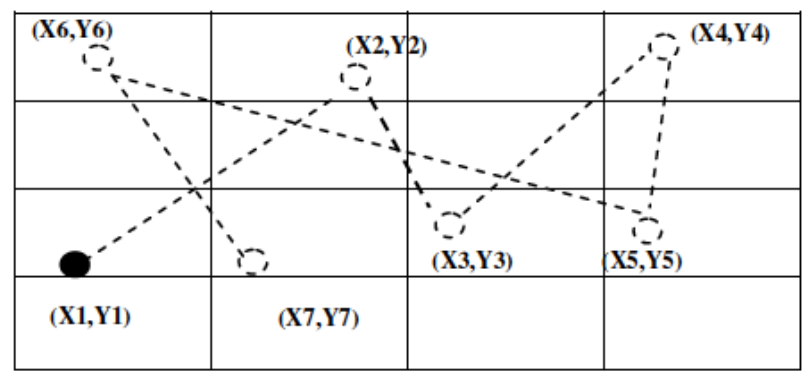

Figura 4. Modelo de movilidad Random Waypoint

Fuente: Tomado de Bano (2010).

Para verificar la funcionalidad multicanal se usó el simulador CRCN (Cognitive Radio Cognitive Network) propuesto en (NSF, 2011). Esta herramienta provee el protocolo Macng, cuya operación se divide en dos fases. Durante la primera, cada nodo envía paquetes con un canal de recepción preferencial. Si hay un canal libre, este se selecciona como el canal preferencial de recepción. En caso contrario, los nodos comparten el canal con el nodo que se encuentra más distante. Durante la segunda fase, el nodo usa el canal seleccionado en la primera fase para enviar y recibir datos. La figura 5 muestra la estructura de diseño que sigue este protocolo.

En cuanto al encaminamiento, las MANETs requieren algoritmos que se adapten rápidamente a los constantes cambios que sufre su topología para mantener la comunicación entre los nodos que la componen. Por esta razón se usó el protocolo de encaminamiento reactivo AODV (Ad Hoc On Demand Distance Vector Routing) (Perkins, 1999).

En las capas de transporte y aplicación se utilizaron los protocolos TCP (Transmission Control Protocol) y FTP (File Transfer Protocol) respectivamente.

\section{RESULTADOS}

El primer experimento que se realizó fue la implementación de la MANET con el protocolo IEEE 802.11 de un solo canal. El desempeño alcanzado bajo estas condiciones se ilustra en la figura 6 . 


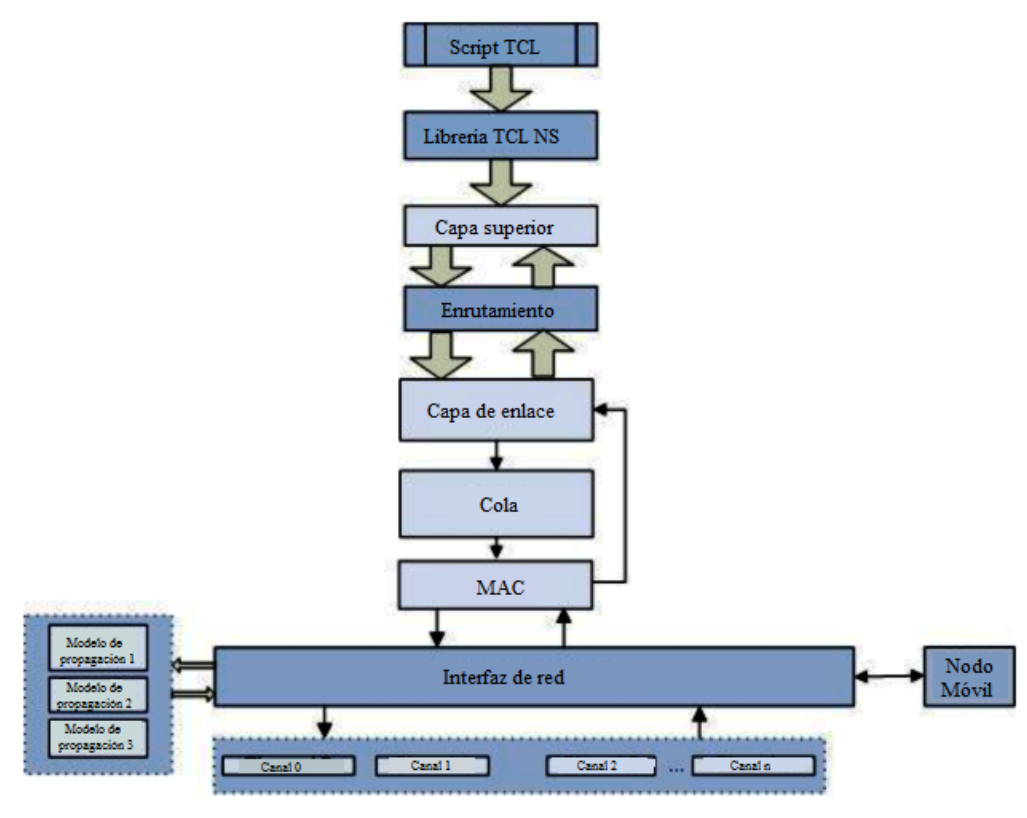

Figura 5. Estructura de diseño del protocolo MMAC-CR.

Fuente: Tomado de NSF (2011).

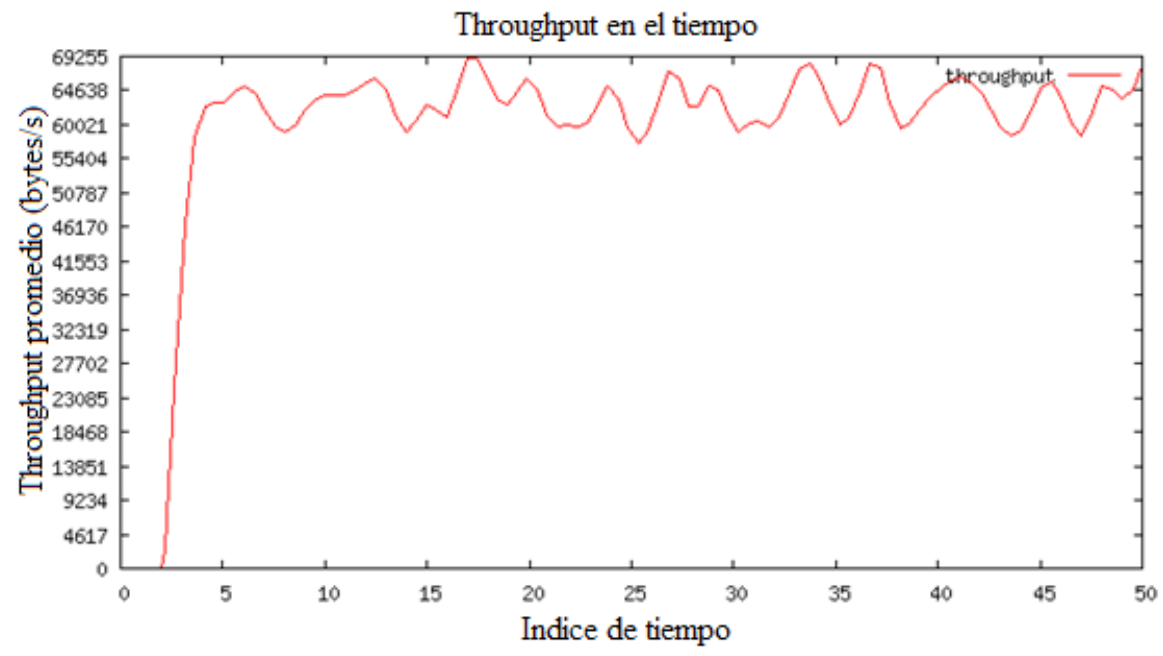

Figura 6. Desempeño de la MANET bajo el protocolo IEEE 802.11 con 1 canal.

Fuente: Elaboración propia.

Luego, se estudió el comportamiento de la red cuando emplea MMAC-CR como protocolo de acceso al medio, pero esta vez utilizando dos canales. El throughput obtenido se ilustra en la figura 7.

La figura 8 presenta el throughput que alcanza la red cuando utiliza tres canales bajo los protocolos IEEE 802.11 y MMAC-CR, respectivamente.

Finalmente, en la figura 9 se exhibe el throughput en la red cuando se usan 4 canales en el protocolo MMAC-CR. 


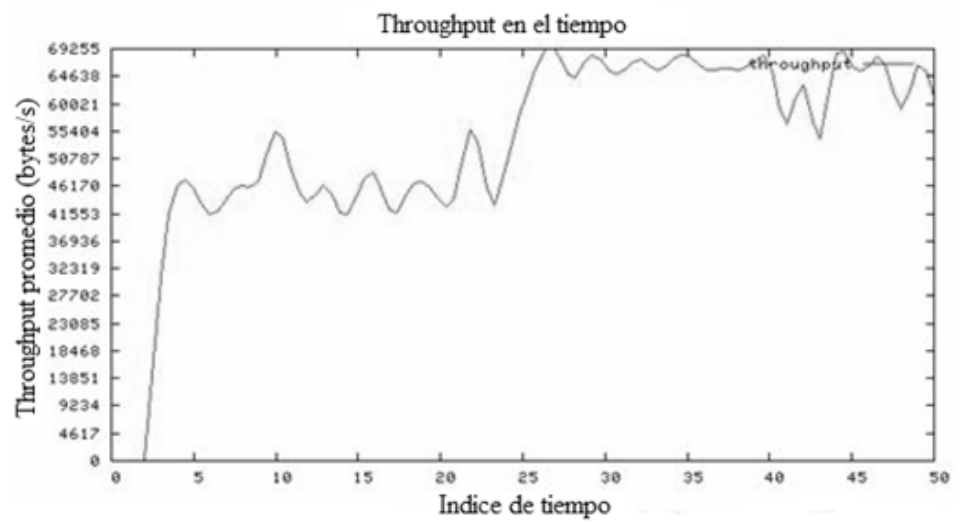

Figura 7. Desempeño de la MANET bajo el protocolo MMAC-CR con 2 canales.

Fuente: Elaboración propia.

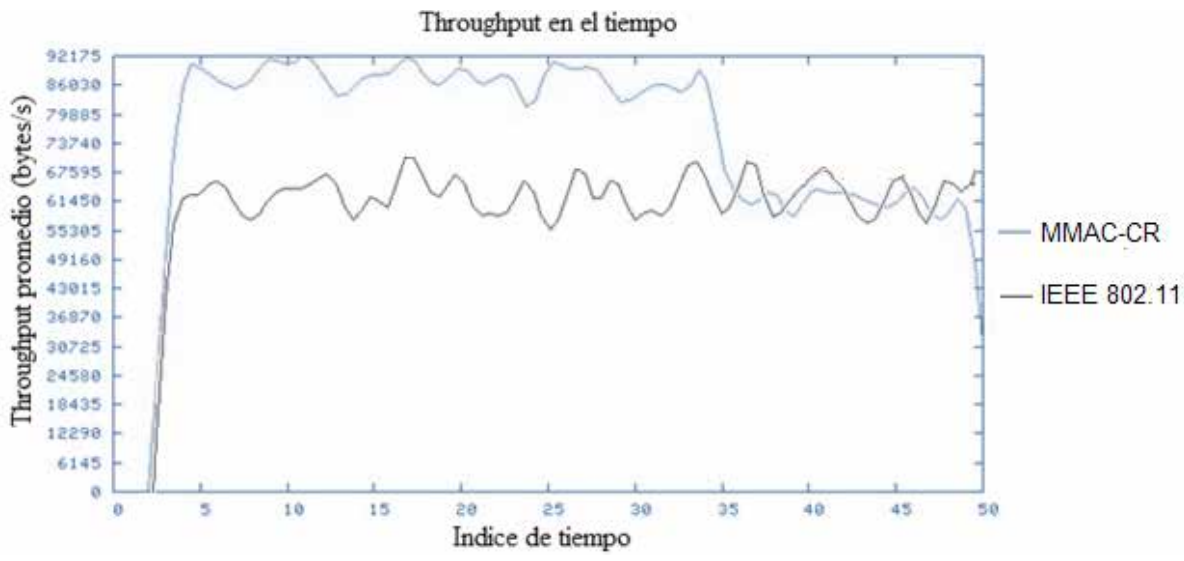

Figura 8. Desempeño de la MANET bajo los protocolos IEEE 802.11 y MMAC-CR con 3 canales.

Fuente: Elaboración propia.

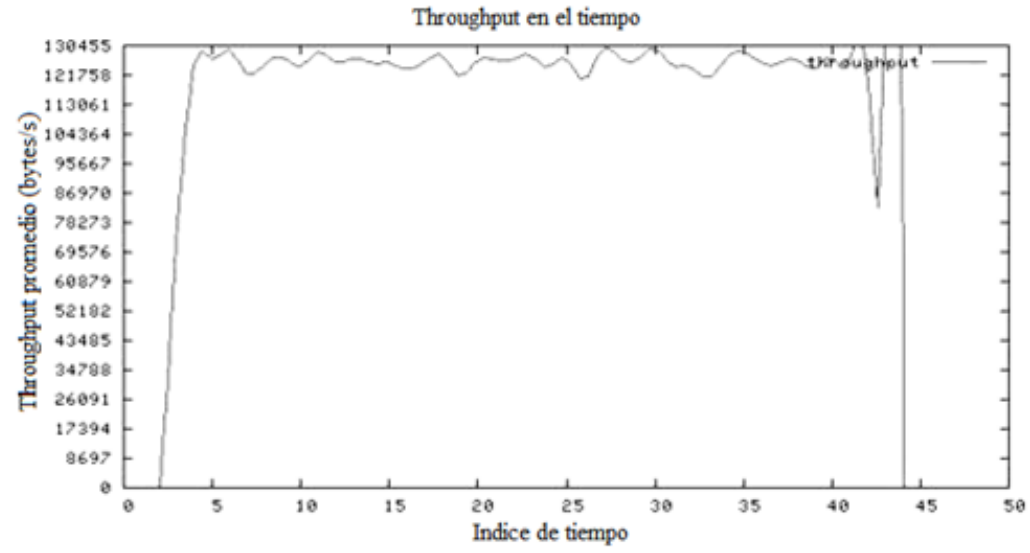

Figura 9. Desempeño de la MANET bajo el protocolo MMAC-CR con 4 canales.

Fuente: Elaboración propia.

Tecnura • p-ISSN: 0123-921X • e-ISSN: 2248-7638• Edición especial 2014 • pp. 125-134 


\section{DISCUSIÓN}

En esta sección se discuten los resultados obtenidos mediante los experimentos realizados.

En primer lugar, comparando la figura 6 y la figura 8 se observa que el desempeño de la red MANET cuando usa IEEE 802.11 con un solo canal es el mismo que cuando usa IEEE 802.11 con múltiples canales. Este resultado es una consecuencia natural del esquema de control de acceso que emplea este protocolo, el cual se fundamenta en la DCF. El diseño de un protocolo MAC que explote múltiples canales bajo este protocolo es un problema complejo, debido a que cada dispositivo IEEE 802.11 está equipado con un transreceptor half-duplex. Aunque el transreceptor es capaz de manejar canales dinámicamente, solo puede transmitir o escuchar sobre un canal cada vez. Entonces, cuando un nodo está escuchando sobre un determinado canal, este no puede escuchar las comunicaciones que tienen lugar sobre los demás canales, causando el problema del terminal oculto multicanal. Por tanto, la operación de un protocolo MAC de un solo canal tal como la DCF de IEEE 802.11 no es óptima en un entorno multicanal en donde los nodos pueden cambiar de canal de manera dinámica (So, 2004).

Por otra parte, el desempeño que alcanza la MANET bajo el protocolo MMAC-CR con dos canales es muy similar al IEEE 802.11, como se presenta en la figura 7, como consecuencia de que uno de estos dos canales se emplea como canal de control común. En la figura 8 se observa la ventaja del aumento a un tercer canal, ya que durante los primeros 35 segundos se mejora el throughput con el protocolo MMAC-CR en aproximadamente $25 \%$, respecto al IEEE 802.11. Esto se debe a que el tercer canal logra compensar el limitado intercambio de datos que se tenía como consecuencia de la dedicación de un canal exclusivo para información de control. Sin embargo, esta mejora solo es relevante, cuando todos los nodos se encuentran dispersos en el área de simulación y la separación entre cada uno de ellos garantiza un nivel mínimo de interferencia entre sus transmisiones.
En otro aspecto, en la figura 8 luego de $35 \mathrm{~s}$ de haberse iniciado la simulación con el protocolo MMAC-CR, se presenta una reducción considerable en el desempeño de la red. Este comportamiento es resultado de las colisiones que se presentan en la red, dada la ubicación de los nodos en el área de simulación, lo que demuestra la gran influencia que tiene la selección del modelo de movilidad en este tipo de redes.

Con la inclusión de un cuarto canal se aprecia una mejoría promedio de alrededor de 50\% del throughput del protocolo MMAC-CR con respecto al IEEE 802.11, tal como se presenta en la figura 9. Solamente a los $44 \mathrm{~s}$ la cantidad de colisiones lleva a que el throughput disminuya considerablemente bajo el protocolo MMAC-CR.

\section{CONCLUSIONES}

En este artículo se ha estudiado el desempeño de los protocolos MAC IEEE 802.11 y MMAC-CR en el contexto de las redes MANET. Para ello se ha desarrollado un script en el que convergen la herramienta de simulación NS-2.31, las mejoras aportadas en (NSF, 2011) y la herramienta de generación de escenarios BonnMotion v1.5a (BonnMotion, 2012).

Los resultados de simulación demuestran que una red MANET alcanza mejores niveles de desempeño bajo el protocolo MMAC-CR que con el protocolo IEEE 802.11, a medida que se aumenta el número de canales. Sin embargo, para pocos canales el protocolo IEEE 802.11 presenta un throughput muy similar al de MMAC-CR para el modelo de movilidad utilizado.

Las funciones básicas de operación del protocolo IEEE 802.11 han sido claves en el desarrollo de protocolos MMAC-CR, permitiendo la coordinación en la transmisión de los paquetes, la reducción de la interferencia y la sincronización en todas las funciones que a nivel de CR maneja la capa MAC.

Como trabajo futuro se propone un estudio comparativo del desempeño de una red MANET bajo diferentes protocolos multicanal, utilizando diferentes modelos de movilidad. 


\section{FINANCIAMIENTO}

\section{Universidad Distrital Francisco José de Caldas}

\section{REFERENCIAS}

Bahl, P.; Chandra R., and Dunagan J. (2004). SSCH: slotted seeded channel hopping for capacity improvement in IEEE 802.11 Ad-Hoc wireless networks. In: Proceedings of MobiCom '04, pp. 216-230.

Bano, N.; Roppel, T., and Gokhale I. (Mar. 2010). Use of mobility models for communication in collaborative robotics. Proceedings of System Theory (SSST), pp. 143-146.

BonnMotion, A mobility scenario generation and analysis tool. http://sys.cs.uos.de/bonnmotion/index.sht$\mathrm{ml}$, Fecha de consulta: marzo 2012.

De Domenico, A.; Strinati, E., and Di Benedetto, M. (2012). A survey on MAC strategies for cognitive radio networks. IEEE Communications Surveys \& Tutorials, vol. 14, No. 1, pp. 21-44.

Federal Comunications Commisions (FCC). Topic 10: License-Exempt wireless applications for public safety. http://transition.fcc.gov/pshs/techtopics/techtopics10.html, Fecha de consulta: mayo de 2012.
Guevara J.; Pedraza L.; Hernández C. Evolución del protocolo MAC multicanal para redes de radio cognitiva. Tecnura, vol. 17, No. 36, 2013, pp.129-139.

Koes, M.; Nourbakhsh I., and Sycara K. Communication efficiency in multi-agent systems. Proceedings of IEEE International Conference on Robotics and Automation (ICRA '04), vol. 3, May 2004, pp. 2129-2134.

National Science Foundation (NSF). Cognitive radio cognitive network simulator. http://faculty.uml.edu/ Tricia_Chigan/Research/CRCN_Simulator.htm, Fecha de consulta: noviembre 2011.

Perkins, C., and Royer, E. Ad-hoc on-demand distance vector routing. Second IEEE Workshop on WMCSA '99, Feb. 1999, pp.90-100.

So, J., and Vaidya, N. Multi-channel MAC for ad hoc networks. Proceedings of MobiHoc '04, 2004, pp. 222-233.

Timmers, M.; Pollin, S.; Dejonghe, A.; Van der Perre L., and Catthoor, F. A distributed multichannel MAC protocol formultihop cognitive radio networks. IEEE Transactions on Vehicular Technology, vol. 59, No. 1, Jan. 2010, pp. 446-459.

\section{(9) $(1) \Theta \Theta$}

